\title{
Theoretical Modeling and Properties of Class DIII Topological Superconductors
}

\author{
Sho Nakosai \\ Department of Applied Physics, University of Tokyo, Tokyo, 113-8656, Japan \\ Yukio Tanaka \\ Department of Applied Physics, Nagoya University, Aichi 464-8603, Japan \\ Naoto Nagaosa \\ RIKEN Center of Emergent Matter Science (CEMS), Wako 351-0198, Japan \\ Department of Applied Physics, University of Tokyo, Tokyo, 113-8656, Japan
}

\begin{abstract}
We theoretically study topological superconductors (TSCs) with time-reversal (TR) symmetry in one- and two-dimensions (1D and 2D). In 2D, we introduce bilayer system with Rashba spin-orbit interaction (SOI), and find that the interplay between SOI and degrees of freedom of layers induces unconventional pairing states, which are topologically nontrivial and have the helical Majorana edge channels. We also find that a usual quantum nanowire on unconventional superconductor becomes a 1D TSC by means of the proximity effect. Majorana fermions at the ends of the wire offer a unique opportunity to relate fermion parity and non-local spin correlations.

Keywords:

Topological Superconductor, Majorana Fermion, Proximity Effect, Nanowire, Spin Correlation
\end{abstract}

\section{Introduction}

The studies on the topological nature of electronic phases have deepened our understanding of the behavior of matter. This perspective originates from the

Preprint submitted to Physica E

August 4, 2013

(C) 2013. This manuscript version is made available under the Elsevier user license http://www.elsevier.com/open-access/userlicense/1.0/ 
pioneering work on the relation between the integer quantum Hall conductance and Chern number by Thouless and collaborators [1]. Through the intensive research on anomalous Hall effect [2] and topological insulators [3, 4], this approach has been revealed to be powerful and fundamental. It is notable that all possible (non-interacting) electronic states can be topologically classified according to their symmetries and spatial dimensions [5, 6, 7]. The concept of TSC has also been proposed in the context of ${ }^{3} \mathrm{He}$ [8], and attracted great interests since TSCs are expected to support Majorana fermions [9, 10, 11, 12, 13, 14. Then there are efforts to implement TSC, especially, in the heterostructures of semiconductor nanowire with Rashba SOI and $s$-wave superconductor under magnetic field [15, 16, 17, 18. The TR symmetry of the system is explicitly broken by magnetic or exchange field in these systems. As a result of topological non-triviality, isolated Majorana fermions appear at the edges and/or the cores of vortices.

Recently, TSCs with the TR symmetry have also been highlighted. They are referred to as DIII TSCs and characterized by $Z_{2}$ number in $1 \mathrm{D}$ and $2 \mathrm{D}$ and by $Z$ number in 3D. The major difference from the above TR breaking TSCs is that the TR symmetry guarantees the Kramers doublet which leads to degenerate Majorana pairs. Theoretical studies suggest the realization of them by using the proximity effect from substrate with non-centrosymmetric superconductors [19, 20], $s_{ \pm}$-wave superconductors [21], and $d$-wave superconductors [22]. Intrinsic superconductivity can also lead to topological phases in $\mathrm{Cu}$ doped $\mathrm{Bi}_{2} \mathrm{Se}_{3}$ [23, 24] and in a theoretical model with two layers of Rashba systems [25]. The physical properties of these systems are now under studies, although several results have already been reported [26, 27].

In this paper, we review our recent works on the 2D [25] and 1D 20] TSCs, whose settings are shown in Figs. 1 (a) and (b), respectively. Both of them are characterized by $Z_{2}$ topological number. The superconducting properties are induced by intrinsic mechanisms and/or the proximity effect. First we introduce the bilayer Rashba system, i.e., two layers of Rashba systems interacting with each other. We will show that SOI and the multi-layer effect induce unconven- 
( a )

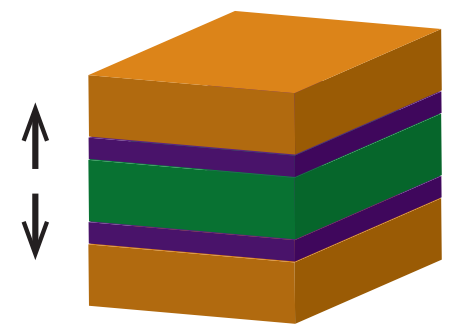

( b )

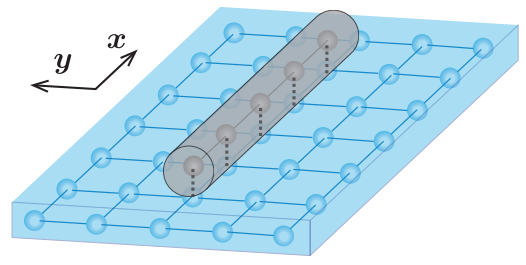

Figure 1: Schematic figures of the systems discussed in the main text. (a) Sandwich structure of oxides shown in brown and green. The blue regions represent $2 \mathrm{D}$ electron gas at the interfaces of oxides while the arrows show the directions of internal electric fields inducing the Rashba SOI. (b) Quantum nanowire on a substrate superconductor with an unconventional pairing. These two are connected through the electron transfer hopping.

tional superconducting pairing. Next, we show the emergence of a topological phase in a quantum nanowire on an unconventional superconductor, and discuss interesting property of resulting Majorana fermions. While the key idea of the previous proposals with breaking TR symmetry is the reduction of electronic degrees of freedom concerning superconductivity, our idea is to explore the spins of Majorana fermions by doubling the degrees of freedom without breaking TR symmetry, which offers richer possibilities.

\section{Bilayer Rashba System}

\subsection{Model}

In this part, we discuss superconductivity in 2D system and its topological nature. The Hamiltonian describing the bilayer Rashba system is given by

$$
H_{0}=\frac{k^{2}}{2 m}-\varepsilon \sigma_{x}+\alpha\left(k_{x} s_{y}-k_{y} s_{x}\right) \sigma_{z},
$$

where $s$ and $\sigma$ denote the Pauli matrices in spin and layer spaces respectively, and $\alpha$ is the strength of Rashba SOI. The second term arises from the hybridization between upper and lower layers with a positive constant $\varepsilon$. We obtain the 


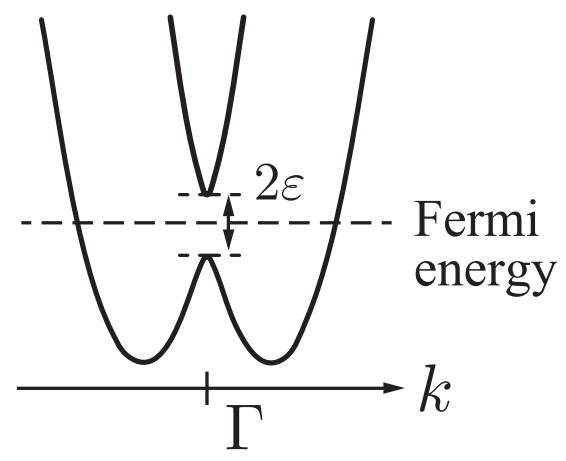

Figure 2: The band structure of the normal state of the Hamiltonian in Eq. 11. There are four bands in total, and each dispersion is doubly degenerate whole through the Brillouin zone. The hybridization causes a gap only at the inner band around $\Gamma$ point.

energy dispersion (Fig. 2)

$$
E_{\boldsymbol{k}}=\frac{k^{2}}{2 m} \pm \sqrt{\varepsilon^{2}+\alpha^{2} k^{2}}=\frac{k^{2}}{2 m} \pm \lambda_{\boldsymbol{k}} .
$$

The band structure is similar to the proposal by Sau and the collaborators [16]. However in the present case the gap at the Dirac point in the inner Fermi surface is induced by hybridization (not by magnetic field), and the bands are doubly degenerate whole through the Brillouin zone due to the TR and inversion symmetries. The key point is that the inversion symmetry is preserved in the total system even though it is locally broken, which leads to the third term with $\sigma_{z}$ in Eq. (1). The $Z_{2}$ topological invariant of TR symmetric TSC can be determined by whether the number of Fermi surfaces enclosing the TR invariant momenta with odd parity pairing potential is odd or even [28, 29, 23. We follow these criteria. In our model it is obvious from the band structure depicted in Fig. 2 that only when the Fermi energy lies within the hybridization gap, topological phase can appear. Next we have to consider when odd parity pairing potential is realized.

Here we mention some experimental results which suggest that an interface system of transition metal oxides provides a good platform for the model 
Eq. (1). As for a single interface, intensive experimental studies of the interface between $\mathrm{SrTiO}_{3}$ and $\mathrm{LaAlO}_{3}$ have revealed that 2D electron gas system is formed there [30, and tunable Rashba SOI [31] as well as superconductivity [32] are observed. It is widely believed that the order parameter in this system is $s$-wave though it is still under discussion. For the determination of the order parameter in our model, we assume that it has four-fold rotational and inversion symmetry with these experimental results in mind. We introduce short-range electron density-density interaction. Note that because we have two layers, there are two kinds of interactions, inter- and intra-layer ones:

$$
\mathcal{H}_{\text {int }}(\boldsymbol{x})=-U\left(n_{1}^{2}(\boldsymbol{x})+n_{2}^{2}(\boldsymbol{x})\right)-2 V n_{1}(\boldsymbol{x}) n_{2}(\boldsymbol{x}),
$$

where $n_{\sigma=1,2}=\sum_{s=\uparrow, \downarrow} c_{\sigma s}^{\dagger} c_{\sigma s}$ is the electron density in layer 1 and $2, U$ and $V$ are introduced as free parameters describing intra-layer and inter-layer interactions, respectively.

\subsection{Results}

The possible pairing potentials are listed in Table 1, and we can determine which order parameter should have the highest critical temperature by considering the gap equations. While $\Delta_{1}$ is ordinary $s$-wave spin-singlet pairing, the others are odd under inversion operation, then, it can lead to topologically nontrivial phase. The effective coupling strength for each $\Delta$ depends on the parameters, especially the magnitude of Rashba SOI. Here we show the explicit equation for each order parameter leading to the phase diagram:

$$
\begin{aligned}
\left|\begin{array}{cc}
U \chi_{0}-1 & U \chi_{01} \\
V \chi_{10} & V \chi_{1}-1
\end{array}\right| & =\left[U+V\left(\varepsilon / \lambda_{\boldsymbol{k}}\right)^{2}\right] \chi_{0}-1=0, \\
V \chi_{2} & =V\left[1-\left(\varepsilon / \lambda_{\boldsymbol{k}}\right)^{2}\right] \chi_{0}=1, \\
U \chi_{3} & =U\left[1-\left(\varepsilon / \lambda_{\boldsymbol{k}}\right)^{2}\right] \chi_{0}=1, \\
V \chi_{4} & =\frac{V}{2}\left[1-\left(\varepsilon / \lambda_{\boldsymbol{k}}\right)^{2}\right] \chi_{0}=1,
\end{aligned}
$$




\begin{tabular}{ccc}
\hline \hline$\Delta_{1}:$ & $c_{1 \uparrow} c_{1 \downarrow}+c_{2 \uparrow} c_{2 \downarrow}, c_{1 \uparrow} c_{2 \downarrow}-c_{1 \downarrow} c_{2 \uparrow}$ & + \\
$\Delta_{2}:$ & $i\left(c_{1 \uparrow} c_{2 \downarrow}+c_{1 \downarrow} c_{2 \uparrow}\right)$ & - \\
$\Delta_{3}:$ & $c_{1 \uparrow} c_{1 \downarrow}-c_{2 \uparrow} c_{2 \downarrow}$ & - \\
$\Delta_{4}:$ & $\left(i\left(c_{1 \uparrow} c_{2 \uparrow}+c_{1 \downarrow} c_{2 \downarrow}\right), c_{1 \uparrow} c_{2 \uparrow}-c_{1 \downarrow} c_{2 \downarrow}\right)$ & - \\
\hline \hline
\end{tabular}

Table 1: The classification of the possible pairings in the model according to the assumed lattice symmetry. The middle column shows the explicit form of the pairing. 1 and 2 denote the upper and lower layer. +/ - signs in the right column are the parity under the inversion operation which exchanges the electrons in a layer and the opposite layer.

where $\chi \mathrm{s}$ are pairing susceptibilities corresponding to channels shown in Table 1 and $\chi_{0}, \chi_{01}$, and $\chi_{1}$ are defined with the Fermi distribution function $f(E)$ as

$$
\begin{aligned}
& \chi_{0}=-\sum_{\boldsymbol{k}}\left[\frac{1-2 f\left(\xi_{\boldsymbol{k}}+\lambda_{\boldsymbol{k}}\right)}{2\left(\xi_{\boldsymbol{k}}+\lambda_{\boldsymbol{k}}\right)}+\frac{1-2 f\left(\xi_{\boldsymbol{k}}-\lambda_{\boldsymbol{k}}\right)}{2\left(\xi_{\boldsymbol{k}}-\lambda_{\boldsymbol{k}}\right)}\right], \\
& \chi_{01}=\chi_{10}=\frac{\varepsilon}{\lambda_{\boldsymbol{k}}} \chi_{0}, \quad \chi_{1}=\left(\frac{\varepsilon}{\lambda_{\boldsymbol{k}}}\right)^{2} \chi_{0} .
\end{aligned}
$$

Since the order parameter belonging to $\Delta_{1}$ in Table 1 is two-dimensional representation and the repulsive inter-layer interaction $V(<0)$ suppresses the second channel, $c_{1 \uparrow} c_{2 \downarrow}-c_{1 \downarrow} c_{2 \uparrow}$, there is a phase transition between $\Delta_{1}$ and $\Delta_{3}$ in the region where $U>0$ and $V<0$. As increasing magnitude of Rashba SOI, the couplings for $\Delta_{i}(i=2,3,4)$ become large. Without SOI, the unconventional superconducting states cannot be the ground state.

Figure 3 is the phase diagram which shows $\Delta$ with the highest critical temperature. Note that this diagram corresponds to the case when the Fermi energy lies within the hybridization gap since it is required for topological phase according to the criteria. So far, we have seen the band structure in the normal state and the phase diagram of the possible pairing. We argue that this system is a promising candidate for TSC. The Fermi energy may be tuned within the hybridization gap in the dilute density case. The intra-layer interaction might be attractive because observed superconductivity is conventional, while the interlayer interaction will be repulsive due to the Coulomb interaction. This case corresponds to $\Delta_{3}$ phase in Fig. 3. Numerical calculation shows the existence of 


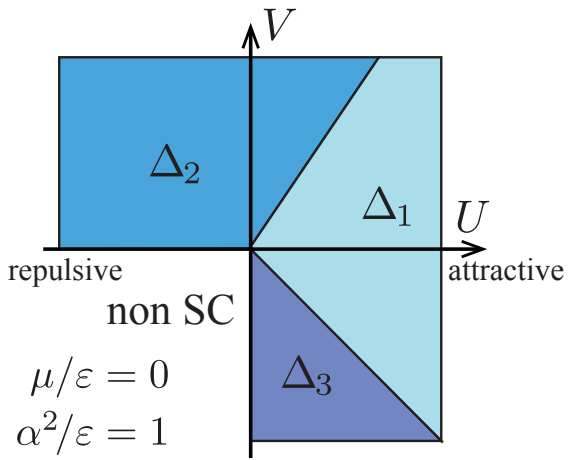

Figure 3: Phase diagram. According to the relation between $U$ and $V$, there are three possibilities. When both of them are attractive, conventional pairing $\Delta_{1}$ is the dominant order over a wide range. On the other hand, when one of them is repulsive SOI induces unconventional pairing $\Delta_{2}$ or $\Delta_{3}$.

mid-gap state only when the criteria for TSC are satisfied. The mid-gap states are doubly degenerate due to the TR symmetry and they are localized at the boundary of the system. We can conclude that they are helical edge states. It is also known that the zero energy states in superconductor are so-called Majorana fermions since they are constructed from electrons and holes with equal weight. Then we call the edge states as helical Majorana states.

\section{Nanowire on Unconventional Superconductor}

\subsection{Model}

Next we consider a nanowire on an unconventional superconductor. As stated in the introductory part, our interest is in TR symmetric cases. We use the proximity effect to the substrate superconductor without Zeeman field. This induces superconducting pairing in the metallic wire. The Hamiltonian 
$H=H_{\mathrm{SC}}+H_{\mathrm{w}}+H_{\mathrm{t}}$ is given as follows:

$$
\begin{aligned}
H_{\mathrm{SC}} & =\int \mathrm{d} k_{x} \mathrm{~d} k_{y} \Psi_{k}^{\dagger}\left[\xi_{\mathrm{SC}}\left(k_{x}, k_{y}\right) \tau_{z}+\Delta_{p} k_{x} \sigma_{z} \tau_{x}-\Delta_{p} k_{y} \tau_{y}\right] \Psi_{k} \\
\Psi_{k}^{\dagger} & =\left(\psi_{k \uparrow}^{\dagger} \psi_{k \downarrow}^{\dagger} \psi_{-k \uparrow} \psi_{-k \downarrow}\right) \\
H_{\mathrm{w}} & =\sum_{\sigma} \int \mathrm{d} k_{x} a_{k_{x} \sigma}^{\dagger}\left[k_{x}^{2} / 2 m_{\mathrm{w}}-\mu_{\mathrm{w}}\right] a_{k_{x} \sigma} \\
H_{\mathrm{t}} & =-c \sum_{\sigma} \int \mathrm{d} x\left(a_{x \sigma}^{\dagger} \psi_{(x, 0) \sigma}+\text { h.c. }\right),
\end{aligned}
$$

where $\sigma$ and $\tau$ denote Pauli matrices in the spin and Nambu spaces, $\xi_{\mathrm{SC}}\left(k_{x}, k_{y}\right)=$ $\left(k_{x}^{2}+k_{y}^{2}\right) / 2 m-\mu$ is the kinetic energy of the substrate, and $c$ is a coupling coefficient between the nanowire and the substrate superconductor. The wire is aligned along $x$-direction as depicted in Fig. 1(b). We have both $s$ - and $p$-wave pairings in general non-centrosymmetric superconductors However topological properties are invariant under the adiabatic deformation $\Delta_{s} \rightarrow 0$ once we started from the case $\left|\Delta_{p}\right|>\left|\Delta_{s}\right|$. Then we assume such a case and only take into account $p$-wave pairing in Eq. 10 .

\subsection{Results}

We are interested in the electronic state in the nanowire rather than the total system. By integrating out the degrees of freedom in the substrate, we obtain an effective model for the 1D system. The integration can be simply performed since $H$ is given in the quadratic form with respect to the operators in the substrate,

$$
\begin{aligned}
G_{\text {eff }}^{-1}\left(i \omega_{n}, k_{x}\right) & =i \omega_{n}-H_{\mathrm{w}}\left(k_{x}\right) \tau_{z}-c^{2} I\left(i \omega_{n}, k_{x}\right), \\
I\left(i \omega_{n}, k_{x}\right) & =\int \mathrm{d} k_{y} \frac{i \omega_{n}+H_{\mathrm{SC}}\left(k_{x}, k_{y}\right)}{\left(i \omega_{n}\right)^{2}-H_{\mathrm{SC}}^{2}\left(k_{x}, k_{y}\right)},
\end{aligned}
$$

where $\omega_{n}$ is Matsubara frequency. We define an effective Hamiltonian from this effective Green's function by taking $\omega_{n}=0$ :

$$
H_{\mathrm{eff}}=2 c^{2} \Delta_{p} k_{x} I_{1} \sigma_{x} \tau_{x}+\left(\xi_{\mathrm{w}}+2 c^{2} \xi_{\mathrm{SC}} I_{1}+2 c^{2} I_{2}\right) \tau_{z},
$$

where $I_{!}$and $I_{2}$ are coefficients determined from Eq. 15) (see Ref. 20] for details). The first term of Eq. (16) is nothing but the induced superconducting 


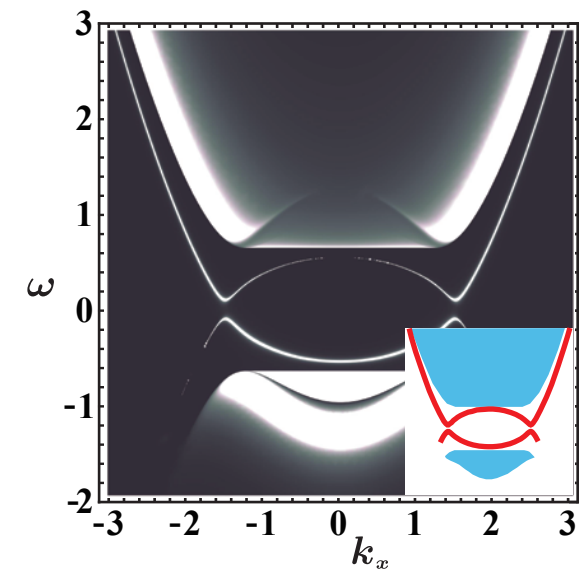

Figure 4: Spectral function of electron Green's function of the effective model. The inset shows the schematic structure of the spectrum. Red lines arise from the nanowire and blue area from the substrate superconductor. The wide gap is the bulk gap in the substrate while the narrow gap at the Fermi energy is the induced gap in the wire.

pairing, and it is in the form of $p$-wave. The spectral function $A\left(\omega, k_{x}\right)$ illustrates the induced superconducting nature clearly, which is given by the retarded Green's function as

$$
\begin{aligned}
G_{\mathrm{eff}}^{\mathrm{R}}\left(\omega, k_{x}\right) & =G_{\mathrm{eff}}\left(\omega+i 0^{+}, k_{x}\right) \\
A\left(\omega, k_{x}\right) & =-\frac{1}{2 \pi} \Im\left[\operatorname{Tr}\left[\tau_{z} G_{\mathrm{eff}}^{\mathrm{R}}\left(\omega, k_{x}\right)\right]\right] .
\end{aligned}
$$

Figure 4 depicts $A\left(\omega, k_{x}\right)$ in Eq. 18), and the inset shows the schematic structure of the spectrum. The red lines come from the metallic nanowire, which have a gap at the Fermi energy induced by the proximity effect to the superconductor. Blue regions correspond to the states in the substrate superconductor. This system is in the topological phase when $|\mu|<|2 D|$, where $\mu$ and $D$ are the chemical potential and the effective band width, respectively. Figure 4 illustrates that case. Numerical calculation with a tight binding model shows that four-fold degenerate zero energy states appear in the topologically nontrivial regime and their wave functions are localized at the ends of the wire. The de- 


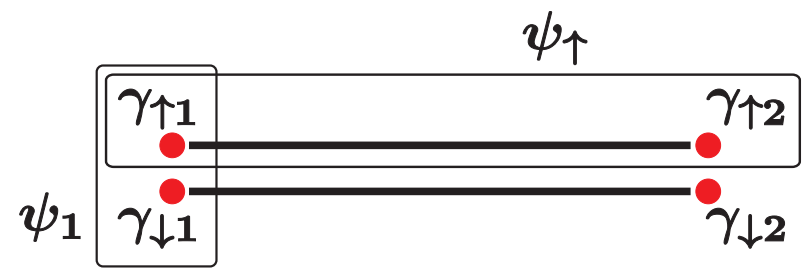

Figure 5: Schematic figure of the system. Black lines represent Kitaev chains, which are TR pairs. They possess Majorana fermions at the ends. $\psi$ s are complex fermions constructed from two Majorana fermions. $\psi_{1}$ is defined from Majorana fermions siting at the same end while $\psi_{\uparrow}$ from ones at both ends.

generacy comes from the TR symmetry. Here we give an intuitive meaning. Our model $H_{\text {eff }}$ can be decoupled into $\sigma_{x}= \pm 1$ spaces. This is because we have no terms which mix the up and down spins. Even when we have them such as SOI, we can decouple $H_{\text {eff }}$ into two subspaces which form TR pairs. Each of them is spinless 1D $p$-wave superconductor known as Kitaev chain. Figure 5 shows the schematic picture of our system which can be regarded as two parallel copies of Kitaev chains. Solid lines represent the chains and circles at the ends are Majorana states. We have four Majorana fermions, $\gamma_{1 \uparrow}, \gamma_{1 \downarrow}, \gamma_{2 \uparrow}$, and $\gamma_{2 \downarrow}$. It is well known that two Majorana fermions constitute one ordinary (complex) fermion: non-local spin polarized ones $\psi_{\uparrow}=1 / 2\left(\gamma_{\uparrow 1}+i \gamma_{\uparrow 2}\right)$ and $\psi_{\downarrow}=1 / 2\left(\gamma_{\downarrow 1}-i \gamma_{\downarrow 2}\right)$, and local ones $\psi_{1}=1 / 2\left(\gamma_{\uparrow 1}+i \gamma_{\downarrow 1}\right)$ and $\psi_{2}=1 / 2\left(\gamma_{\uparrow 2}-i \gamma_{\downarrow 2}\right)$ in our system. We focus on one end, where two Majorana fermions are sitting. We introduce spin operator following the definition $\boldsymbol{s}_{1(2)}=1 / 2 \times \gamma_{1(2) \alpha} \boldsymbol{\sigma}_{\alpha \beta} \gamma_{1(2) \beta}$. Due to the special property of Majorana states, their antiparticles are equal to themselves, $x$ and $z$ components of the operator are identical to 0 . Therefore Ising-like spin sits on each end of the wire. Fermion parity is commonly used to characterize systems of Majorana states, which measures even or odd numbers of fermions in the quantum system [10, 33, 34]. Now we consider the relationship between spins defined above and fermion parity. We calculate the correlation of two spins $\boldsymbol{s}_{1}$ and $\boldsymbol{s}_{2},\left\langle s_{1 y} s_{2 y}\right\rangle$. To deal with the problem, we assume that the to- 
tal system is a mesoscopic system, and introduce so-called charging energy and gate voltage. This charging energy causes the difference in energies according to fermion parity. The ground state is originally four-fold degenerate since we have four Majorana states. After switching on charging energy these four split into two doubly degenerate spaces, even and odd. The density matrices in the thermal equilibrium state are given by $\rho_{\text {odd }}=\frac{1}{2}\left(|0\rangle_{1}|1\rangle_{21}\left\langle\left. 0\right|_{2}\langle 1|+| 1\rangle_{1} \mid 0\right\rangle_{21}\left\langle\left. 1\right|_{2}\langle 0|\right)\right.$ and $\rho_{\text {even }}=\frac{1}{2}\left(|0\rangle_{1}|0\rangle_{21}\left\langle\left. 0\right|_{2}\langle 0|+| 1\rangle_{1} \mid 1\right\rangle_{21}{ }_{1}\left\langle\left. 1\right|_{2}\langle 1|\right)\right.$, respectively. Here $|0\rangle_{1}$ and $|1\rangle_{1}$ are states satisfying $\psi_{1}|0\rangle_{1}=0$ and $|1\rangle_{1}=\psi_{1}|0\rangle_{1}$. We find that in each space the correlation has finite value and they are different by sign with each other:

$$
-\left\langle s_{1 y} s_{2 y}\right\rangle_{\text {odd }}=\left\langle s_{1 y} s_{2 y}\right\rangle_{\text {even }}=\frac{1}{4}
$$

where $\langle\ldots\rangle_{\text {odd } / \text { even }}$ represents the statistical average with the density matrices $\rho_{\text {odd }}$ and $\rho_{\text {even }}$, respectively. These results show that we can control the nonlocal spin-spin correlation by modulating charging energy and the gate voltage. This is independent of the distance of spins since they are, by the construction, connected through the superconducting wire in between. It is a surprising result, though it is a consequence of non-locality of Majorana fermions.

\section{Conclusions}

In this article, we reviewed (i) the search for topological superconductivity in the interacting bilayer Rashba systems, which can be realized at the interfaces of oxides, and (ii) the proximity effect of a nanowire without Zeeman field on an unconventional superconductor. In both cases, the resulting topological states have the TR symmetry, and Majorana states appear accompanied by their TR partners. In the bilayer system, the degrees of freedom of layers supply various possibilities of order parameter, and SOI favors unconventional ones. Since the resulting Majorana states are degenerate, we regard them as spinful Majorana states. The correlation between two spins at the opposite ends of the wire can be controlled by electric means due to the non-locality of Majorana states. 


\section{Acknowledgments}

S. N. was supported by Grant-in-Aid for JSPS Fellows. We appreciate our collaborators, J. C. Budich and B. Trauzettel. This work was supported by Grant-in-Aid for Scientific Research (S) (Grant No. 24224009); the Funding Program for World-Leading Innovative RD on Science and Technology (FIRST Program); Strategic International Cooperative Program (Joint Research Type) from Japan Science and Technology Agency; Innovative Areas "Topological Quantum Phenomena" (Grant No. 22103005) from the Ministry of Education, Culture, Sports, Science, and Technology of Japan.

\section{References}

[1] D. J. Thouless, M. Kohmoto, M. P. Nightingale, M. den Nijs, Phys. Rev. Lett. 49 (1982) 405-408.

[2] N. Nagaosa, J. Sinova, S. Onoda, A. H. MacDonald, N. P. Ong, Rev. Mod. Phys. 82 (2010) 1539-1592.

[3] M. Z. Hasan, C. L. Kane, Rev. Mod. Phys. 82 (2010) 3045-3067.

[4] X.-L. Qi, S.-C. Zhang, Rev. Mod. Phys. 83 (2011) 1057-1110.

[5] A. P. Schnyder, S. Ryu, A. Furusaki, A. W. W. Ludwig, Phys. Rev. B 78 (2008) 195125.

[6] S. Ryu, A. P. Schnyder, A. Furusaki, A. W. W. Ludwig, New J. Phys. 12 (2010) 065010.

[7] A. Kitaev, AIP Conf. Proc. 1134 (2009) 22-30.

[8] G. R. Volovik, The Universe in a Helium Droplet, Oxford Science Publications, 2003.

[9] N. Read, D. Green, Phys. Rev. B 61 (2000) 10267-10297.

[10] A. Y. Kitaev, Phys. Usp. 44 (2001) 131. 
[11] D. A. Ivanov, Phys. Rev. Lett. 86 (2001) 268-271.

[12] M. Stone, R. Roy, Phys. Rev. B 69 (2004) 184511.

[13] L. Fu, C. L. Kane, Phys. Rev. Lett. 100 (2008) 096407.

[14] J. Linder, Y. Tanaka, T. Yokoyama, A. Sudbø, N. Nagaosa, Phys. Rev. Lett. 104 (2010) 067001.

[15] M. Sato, Y. Takahashi, S. Fujimoto, Phys. Rev. Lett. 103 (2009) 020401.

[16] J. D. Sau, R. M. Lutchyn, S. Tewari, S. Das Sarma, Phys. Rev. Lett. 104 (2010) 040502.

[17] J. Alicea, Phys. Rev. B 81 (2010) 125318.

[18] Y. Oreg, G. Refael, F. von Oppen, Phys. Rev. Lett. 105 (2010) 177002.

[19] C. L. M. Wong, J. Liu, K. T. Law, P. A. Lee, arXiv:1206.5601 (2012).

[20] S. Nakosai, J. C. Budich, Y. Tanaka, B. Trauzettel, N. Nagaosa, Phys. Rev. Lett. 110 (2013) 117002.

[21] F. Zhang, C. L. Kane, E. J. Mele, arXiv:1212.4232 (2012).

[22] C. L. M. Wong, K. T. Law, Phys. Rev. B 86 (2012) 184516.

[23] L. Fu, E. Berg, Phys. Rev. Lett. 105 (2010) 097001.

[24] S. Sasaki, M. Kriener, K. Segawa, K. Yada, Y. Tanaka, M. Sato, Y. Ando, Phys. Rev. Lett. 107 (2011) 217001.

[25] S. Nakosai, Y. Tanaka, N. Nagaosa, Phys. Rev. Lett. 108 (2012) 147003.

[26] A. Yamakage, K. Yada, M. Sato, Y. Tanaka, Phys. Rev. B 85 (2012) 180509.

[27] X.-J. Liu, C. L. M. Wong, K. T. Law, arXiv:1304.3765 (2013).

[28] X.-L. Qi, T. L. Hughes, S.-C. Zhang, Phys. Rev. B 81 (2010) 134508.

[29] M. Sato, Phys. Rev. B 81 (2010) 220504. 
[30] A. Ohtomo, H. Y. Hwang, Nature 427 (2004) 423-426.

[31] K. Michaeli, A. C. Potter, P. A. Lee, Phys. Rev. Lett. 108 (2012) 117003.

[32] N. Reyren, S. Thiel, A. D. Caviglia, L. F. Kourkoutis, G. Hammerl, C. Richter, C. W. Schneider, T. Kopp, A.-S. Retschi, D. Jaccard, M. Gabay, D. A. Muller, J.-M. Triscone, J. Mannhart, Science 317 (2007) 1196-1199.

[33] L. Fu, C. L. Kane, Phys. Rev. B 79 (2009) 161408.

[34] L. Fu, Phys. Rev. Lett. 104 (2010) 056402. 